\title{
Dosimetric comparison between interstitial brachytherapy and volumetric-modulated arc therapy for tumor bed boost in breast cancer
}

\author{
Dr. Kannan Periasamy, MD, DNB, Assist. Prof.'.2, Dr. Gunaseelan Karunanithi, MD, Addit. Prof.', \\ Dr. Shamsudheen Cholayil, MD³, Dr. Prof. Kadambari Dharanipragada, MS 4 , \\ Dr. Vijayprabhu Neelakanadan, MSc, PhD, Assist. Prof.2 \\ 'Department of Radiotherapy, PGIMER, Chandigarh, India, ${ }^{2}$ Department of Radiation Oncology, Regional Cancer Centre, JPMER, \\ Puducherry, India, ${ }^{3}$ MVR Cancer Centre, Kozhikode, India, ${ }^{4}$ Department of General Surgery, JPMER, Puducherry, India \\ The work was conducted in Department of Radiation Oncology, Regional Cancer Centre, JPMER, Puducherry, India.
}

\begin{abstract}
Purpose: To dosimetrically compare high-dose-rate interstitial brachytherapy (HDR-BT) with volumetric-modulated arc therapy (VMAT) for tumor bed boost, following breast conservative treatment.

Material and methods: 50 patients with early-stage breast cancer who underwent breast conservation surgery, followed by either HDR-BT $(n=25)$ of 15 Gy in 6 fractions over a period of 3 days, or VMAT dose of 16 Gy in 8 fractions $(n=25)$ for tumor bed boost, were retrospectively reviewed. All patients received whole breast irradiation of $46 \mathrm{~Gy}$ in 23 fractions. Dosimetric parameters for organs at risk (OARs), including ipsilateral and contralateral lungs, heart, contralateral breast, skin, and ribs, were evaluated with the help of dose-volume histograms (DVH).

Results: Heart sparing was similar in both modalities (left-sided breast irradiation, HDR-BT $\mathrm{D}_{2 \mathrm{cc}} 20.5 \%$ vs. VMAT $30.2 \%, p$-value $=0.243$; right-sided breast irradiation, $\mathrm{D}_{2 c c} 6.5 \%$ vs. $4.4 \%, p$-value $\left.=0.165\right)$. Left-sided cases received higher dose to heart compared to right-sided patients. Interstitial brachytherapy resulted in significantly less dose to contralateral breast $\left(\mathrm{D}_{2 c \mathrm{cc}} 4.3 \%\right.$ vs. 9.6\%, p-value $\left.<0.0001\right)$, ipsilateral lung $\left(\mathrm{D}_{2 c \mathrm{cc}} 27.6 \%\right.$ vs. $73.2 \%, p$-value $\left.<0.0001\right)$, contralateral lung $\left(\mathrm{D}_{2 \mathrm{cc}} 4.2 \%\right.$ vs. $14.5 \%, p$-value $\left.<0.0001\right)$, ribs $\left(\mathrm{D}_{2 \mathrm{cc}} 24.1 \%\right.$ vs. $41.2 \%, p$-value $\left.<0.0001\right)$, and skin $\left(\mathrm{D}_{2 \mathrm{cc}}\right.$ $77.3 \%$ vs. $95 \%, p$-value $<0.0001$ ).

Conclusions: HDR-BT-based tumor bed boost irradiation results in significantly lower doses to most organs at risk with similar heart sparing compared to VMAT.

J Contemp Brachytherapy 2021; 13, 3: 302-309 DOI: https://doi.org/10.5114/jcb.2021.106141
\end{abstract}

Key words: breast neoplasms, radiotherapy, volumetric-modulated arc therapy, interstitial brachytherapy.

\section{Purpose}

Global Cancer Data, GLOBOCAN 2018, has estimated that breast cancer is the most common cancer, representing approximately $14 \%$ of total cancer cases in India. It is the leading cause of cancer mortality, accounting for $11.1 \%$ of the total cancer-related deaths [1]. Breast conservative therapy (BCT), the standard of care for early breast cancer, involves lumpectomy with an adequate margin and axillary clearance, followed by adjuvant radiotherapy. It has been proven in multiple randomized control trials that there is no difference in survival outcomes between breast conservative treatment and total mastectomy [2, 3]. Adjuvant radiotherapy, following breast conservative surgery, involves irradiation of the whole breast as well as regional lymphatics in high-risk cases [4]. The recurrence in the vicinity of tumor bed was
$50-60 \%$ of all local recurrences, which necessitated tumor bed boost irradiation $[5,6]$. Whole breast irradiation to a dose of 45-50 Gy and a tumor bed boost of 10-20 Gy are recommended for adjuvant treatment following breast conservative surgery in patients with high-risk features (age $<50$ years, grade 3 tumors, extensive intraductal component) [7]. The benefit of boost irradiation has been clearly shown in the European Organization for Research and Treatment of Cancer (EORTC) trial, which demonstrated a significant improvement in local control, especially among younger patients who received a tumor bed boost irradiation [8]. Various techniques used for delivering tumor bed boost include conformal teletherapy with fixed electron or photon beams, intensity-modulated radiation therapy (IMRT), volumetric-modulated arc therapy (VMAT), and interstitial brachytherapy.
Address for correspondence: Dr. Gunaseelan Karunanithi, MD, Addit. Prof., Department of Radiation Oncology, Regional Cancer Centre, JIPMER, Puducherry-605006, India, phone: +93 62966444, $\bowtie$ e-mail: gunapgi@gmail.com
Received: 01.10 .2020

Accepted: 08.04.2021

Published: 14.05.2021 
High-dose-rate brachytherapy (HDR-BT), despite being invasive, is a well-studied modality for partial breast irradiation as well as for tumor bed boost after breast conservative surgery in breast cancers [9-18]. Volumetric-modulated arc therapy is a novel non-invasive radiation treatment technique that is based on volumetric-modulated rotational delivery, as compared to classic IMRT, which uses fixed gantry beams. By varying the speed of gantry rotation, multi-leaf collimator shape and continuously changing fluence (dose-rate), the VMAT delivers highly conformal IMRT plans in a short time, with less monitors units and possibly less dose to organs at risk [19]. The choice of boost technique is very important in breast cancer radiotherapy. Although dosimetric comparisons between brachytherapy and 3-dimensional conformal radiotherapy (3D-CRT) and IMRT were available [20-22], there is a lack of data on comparison of incidental doses to organs at risk (OARs) with tumor bed boost irradiation by VMAT and brachytherapy. Hence, the purpose of the study was to dosimetrically compare organs at risk between interstitial brachytherapy and VMAT-based tumor bed boost, following breast conservative surgery.

\section{Material and methods}

Treatment planning data of 50 patients with breast cancer who underwent breast conservation surgery, followed by either HDR interstitial brachytherapy $(n=25)$ or VMAT $(n=25)$ for tumor bed boost at regional cancer center, Jawaharlal Institute of Postgraduate Medical Education and Research Institute (JIPMER), were included in this retrospective study. All patients received whole breast irradiation of $46 \mathrm{~Gy}$ in 23 fractions. Tumor bed boost was considered in all patients $<50$ years of age or those with high-risk features, such as grade 3 tumors and/or extensive intraductal component. No specific criteria were considered for choosing the technique for tumor bed boost. The organs at risk evaluated included the ipsilateral and contralateral lungs, heart, ipsilateral and contralateral breasts, skin, and ribs. All these organs at risk were contoured using available contouring atlases $[23,24]$. Treatment planning for both groups were performed to meet the criteria provided by the Radiation Therapy Oncology Group (RTOG) 0413 protocol for target coverage and OARs sparing [25].

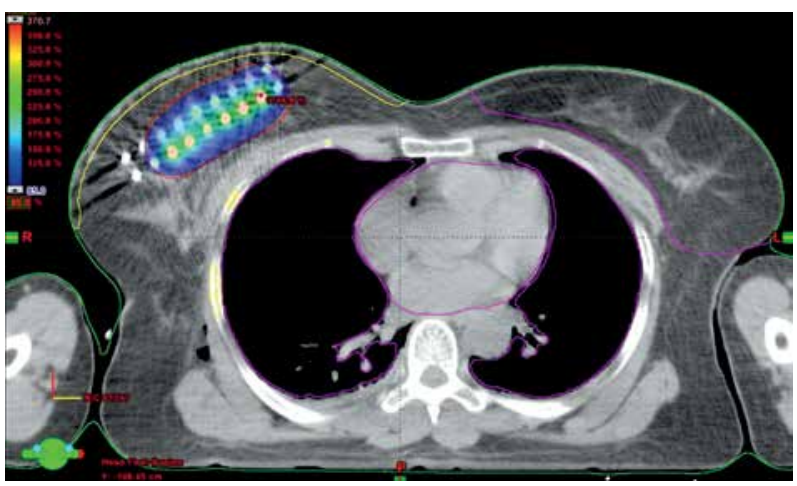

Fig. 1. Dose color wash in rigid implant

\section{Interstitial brachytherapy}

The patients in the brachytherapy group received perioperative interstitial HDR-BT of 15 Gy in 6 fractions over a period of 3 days as a boost, and whole breast irradiation was delivered after a gap of 2-3 weeks. Stainless steel or flexible catheters were implanted on operating table after lumpectomy and axillary dissection. Catheters were implanted after inspection of the tumor bed with guidance of template, so as to maintain the inter-catheter distance of $1 \mathrm{~cm}$ and preserve the geometry of the implant. The number of planes and number of catheters were decided intra-operatively, after examining the tumor bed and assessing the remaining breast volume. These patients were simulated after 24-48 hours in computed tomography (CT) simulator, and a $3 \mathrm{~mm}$ slice CT images were obtained from lower neck to upper abdomen; the images were then transferred to Oncentra treatment planning system (Elekta Instrument, AB Stockholm, Sweden). Target delineation was performed according to the GEC-ESTRO Breast Cancer Working Group recommendations [26]. The clinical target volume (CTV) volume included lumpectomy cavity plus at least $1-2 \mathrm{~cm}$ margin, depending on the size of the resection margin and cropped from the underlying pectoralis muscle and overlying skin by $5 \mathrm{~mm}$. Skin was contoured as $5 \mathrm{~mm}$ thickness from the body surface. As there is no margin given for uncertainties in brachytherapy, planning target volume (PTV) became the CTV. The catheters were reconstructed and dose optimization was performed by adjusting dwell times in individual dwell positions in order to achieve the target volume coverage. AAPM TG43 formalism was used to generate dose distributions. Source position nearest to skin and ribs were placed at a distance of $10 \mathrm{~mm}$ to decrease high-dose regions within the ribs and skin. The dosimetric parameters evaluated included target coverage $\left(\mathrm{V}_{90}, \mathrm{D}_{90}\right)$, high-dose volumes $\left(\mathrm{V}_{150}, \mathrm{~V}_{200}\right)$, dose homogeneity index $(\mathrm{DHI})$, where $\mathrm{DHI}=\left(1-\mathrm{V}_{150} / \mathrm{V}_{100}\right)$, and maximum skin isodose. It was aimed to maintain $\mathrm{V}_{90}>90 \%$ of the PTV volume and $\mathrm{D}_{90}>90 \%$ of the prescribed dose as well as to maintain the skin $\mathrm{D}_{1 \mathrm{cc}}<80-90 \%$. Figure 1 shows dose color wash in a patient with twoplane rigid implant.

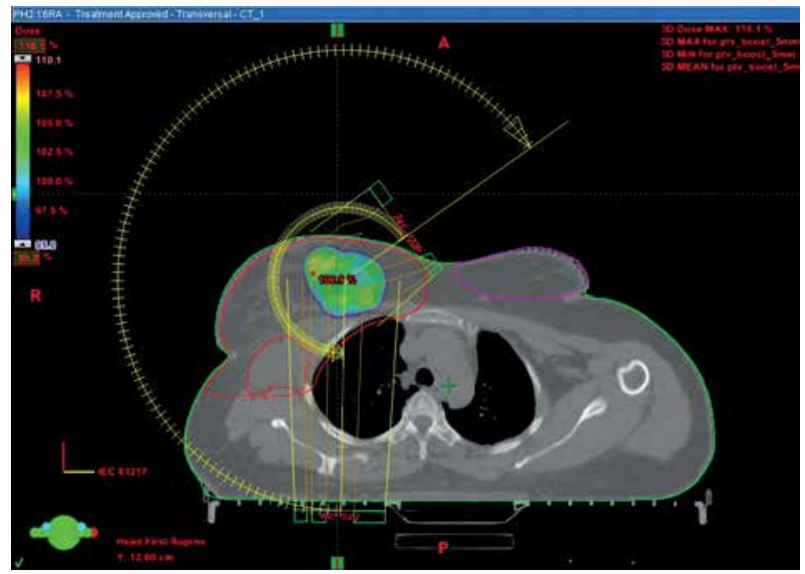

Fig. 2. VMAT dose distribution - dose covered with $95 \%$ of the prescribed dose 


\section{Volumetric-modulated arc therapy}

In the VMAT group, boost irradiation was applied after completion of whole breast irradiation. Usually, no interval was given between whole breast irradiation and boost irradiation. The seroma was contoured in CT planning obtained for whole breast radiation therapy (RT), with clips also contoured as a separate structure. Subsequently, CTV boost was created by adding the seroma and clips and by adding a margin of $1 \mathrm{~cm}$, so that the clips were completely included in the CTV with a margin for microscopic disease. The CT planning was then registered with the pre-operative $\mathrm{CT}$, and the location of previous tumor and tumor bed in the CT planning were confirmed. A margin of $5 \mathrm{~mm}$ was given for PTV boost from CTV boost but it was cropped to lie $5 \mathrm{~mm}$ below the skin. PTV boost_eval was defined by cropping PTV boost $5 \mathrm{~mm}$ inside the skin surface. PTV

Table 1. Patient, tumor, and treatment characteristics

\begin{tabular}{|c|c|c|c|c|}
\hline \multirow[t]{2}{*}{ Variable } & \multicolumn{2}{|c|}{ VMAT } & \multicolumn{2}{|c|}{ Brachytherapy } \\
\hline & No. & $\%$ & No. & $\%$ \\
\hline No. of patients & \multicolumn{2}{|c|}{25} & \multicolumn{2}{|c|}{25} \\
\hline Median age (years) & \multicolumn{2}{|c|}{45} & \multicolumn{2}{|c|}{48} \\
\hline \multicolumn{5}{|l|}{ Side } \\
\hline Left & 15 & 60 & 16 & 64 \\
\hline Right & 10 & 40 & 9 & 36 \\
\hline \multicolumn{5}{|l|}{ Site of the tumor } \\
\hline Upper outer & 14 & 56 & 10 & 40 \\
\hline Upper inner & 5 & 20 & 11 & 44 \\
\hline Lower inner & 2 & 8 & 1 & 4 \\
\hline Lower outer & 4 & 16 & 3 & 12 \\
\hline \multicolumn{5}{|l|}{ T size } \\
\hline $\mathrm{T} 1$ & 4 & 16 & 0 & 0 \\
\hline $\mathrm{T} 2$ & 12 & 48 & 22 & 88 \\
\hline T3 & 9 & 36 & 3 & 12 \\
\hline \multicolumn{5}{|l|}{$\mathrm{N}$ status } \\
\hline NO & 14 & 56 & 17 & 68 \\
\hline N1 & 11 & 44 & 8 & 32 \\
\hline \multicolumn{5}{|l|}{ Stage } \\
\hline 1 & 4 & 16 & 0 & 0 \\
\hline 2 & 17 & 68 & 24 & 96 \\
\hline 3 & 4 & 16 & 1 & 4 \\
\hline \multicolumn{5}{|l|}{ Receptor status } \\
\hline ER positive & 13 & 52 & 14 & 56 \\
\hline PR positive & 7 & 28 & 8 & 32 \\
\hline Her 2 neu positive & 10 & 40 & 11 & 44 \\
\hline \multicolumn{5}{|l|}{ Chemotherapy } \\
\hline FAC & 12 & 48 & 15 & 60 \\
\hline FEC + docetaxel & 11 & 44 & 9 & 36 \\
\hline Docetaxel + carboplatin & 1 & 4 & 0 & 0 \\
\hline No chemotherapy & 1 & 4 & 1 & 4 \\
\hline
\end{tabular}

boost_eval was used for generating dose-volume histograms (DVHs) and comparative analyses. It was aimed to keep $\mathrm{V}_{90}>90 \%$ of the PTV boost_eval and $\mathrm{D}_{90}>90 \%$ of the prescribed dose. VMAT plans were created using Eclipse (version 10, Varian Medical Systems, USA) treatment planning system (TPS) using $6 \mathrm{MV}$ photon arcs. The plans were made with one or two partial arcs, avoiding direct entrance through the contra-lateral breast by applying angulation to treatment couch. The dose schedule for VMAT was $16 \mathrm{~Gy}$ in 8 fractions. Figure 2 demonstrates dose distribution in a patient treated with VMAT-based tumor bed boost.

\section{Statistical analysis}

Patient and tumor characteristics were expressed as frequencies and percentages. SPSS software was utilized to perform the statistical analysis. Shapiro-Wilk's test $(p<0.05)$ and visual inspection of histograms, Q-Q plots, and box plots revealed that the dosimetric data were not normally distributed for both the groups. Therefore, the data were expressed as a median with interquartile range. A non-parametric test (Mann-Whitney $U$ ) was applied for the analysis of parameters between the groups. A $p$-value of $<0.05$ was considered significant.

\section{Results \\ Characteristics}

The patient and tumor characteristics are summarized in Table 1. The general characteristics of patients in each group were comparable. This study included T1, T2, and T3 tumors, which were down-staged by neoadjuvant chemotherapy. Out of the 25 patients in the brachytherapy group, 22 were rigid implants with a template and three were flexible implants. The median number of planes was 2, sixteen cases were two-plane implant, six cases were three-plane implant, and three cases were singleplane, as indicated in Table 2.

\section{Target volumes}

The results of brachytherapy and VMAT target volume parameters are presented in Table 3 as median and interquartile ranges $\left(25^{\text {th }}\right.$ quartile and $75^{\text {th }}$ quartile). In the brachytherapy group, the median PTV volume was $92.4 \mathrm{cc}$ (range, 63.7-119.2 cc); $\mathrm{D}_{90}$ and $\mathrm{V}_{90}$ were 92.1\% (range, $78.2-104.5 \%$ ) of the prescribed dose and 91.3\% (range, 82-97\%) of the PTV volume, respectively;

Table 2. Brachytherapy

Variable

No. of cases

\begin{tabular}{lc}
\hline No. of planes & \\
\hline 1 & 16 \\
\hline 2 & 6 \\
\hline 3 & 22 \\
\hline Type of implant & 3 \\
\hline Rigid implant & $17(8-24)$
\end{tabular}


Table 3. Target volume parameters

Brachytherapy PTV parameters $\quad$ VMAT PTV parameters

\begin{tabular}{lccccc}
\hline Dosimetric parameter & Median & IQR & Dosimetric parameter & Median & IQR \\
\hline PTV volume & $92.4 c c$ & $63.7-119.2$ & PTV volume & $133.0 c c$ & $75.1-158.0$ \\
\hline$D_{90}$ & $92.1 \%$ & $78.2-104.5$ & $D_{2 \%}$ & $106.1 \%$ & $104.5-108.9$ \\
\hline$V_{90}$ & $91.3 \%$ & $82.0-97.0$ & $D_{98 \%}$ & $97.3 \%$ & $90.4-100.8$ \\
\hline$V_{100}$ & $84 \%$ & $74.0-93.0$ & $D_{90 \%}$ & $99.9 \%$ & $97.7-110.7$ \\
\hline$V_{150}$ & $44 c c$ & $18.5-70.0$ & $D_{95 \%}$ & $99.3 \%$ & $95.2-103.5$ \\
\hline$V_{200}$ & $21 c c$ & $10.7-31.0$ & $D_{\max }$ & $108.4 \%$ & $106.0-112.3$ \\
\hline$V_{300}$ & $7.4 c c$ & $4.2-12.2$ & $D_{\min }$ & $89.6 \%$ & $70.0-94.0$ \\
\hline DNR & 0.45 & $0.32-0.61$ & $D_{\operatorname{mean}}$ & $103.1 \%$ & $101.0-105.7$ \\
\hline DHI & 0.55 & $0.39-0.68$ & $\mathrm{D}_{\operatorname{median}}$ & $103.3 \%$ & $102.0-105.9$ \\
\cline { 3 - 5 } & & & $\mathrm{V}_{90}$ & $99.9 \%$ & $99.8-100.0$ \\
\hline Coverage index & 0.84 & $0.74-0.93$ & $\mathrm{~V}_{100}$ & 89.9 & $79.6-99.8$ \\
& & & $\mathrm{Cl}$ & 0.94 & $0.81-1.1$ \\
\hline
\end{tabular}

VMAT - volumetric-modulated arc therapy, PTV - planning target volume, DNR - dose non-uniformity ratio, DHI-dose homogeneity index, CI - conformity index, $\mathrm{HI}$ - homogeneity index

$\mathrm{V}_{100}$ was $84 \%$ (range, 74-93\%). High-dose volumes, $\mathrm{V}_{150}$ and $\mathrm{V}_{200}$ were $44 \mathrm{cc}$ (range, 18.5-70 cc) and $21 \mathrm{cc}$ (range, $10.7-31 \mathrm{cc})$, respectively. The median DHI $\left(1-\mathrm{V}_{150} / \mathrm{V}_{100}\right)$ was 0.55 (range, 0.39-0.68). In the VMAT group, the median PTV volume was 133 cc (range, 75.1-158 cc); $\mathrm{D}_{90}$ was 99.9\% (range, $97.7-110.7 \%$ ) of the prescribed dose and $\mathrm{D}_{\max }$ was $108.4 \%$ (range, $106-112.3 \%$ ); $\mathrm{V}_{90}$ and $\mathrm{V}_{100}$ were 99.9\% (range, 99.8-100\%) and 89.9\% (range, 79.6-99.8\%) of the PTV volume, respectively. The median conformity $(\mathrm{CI}=$ treated volume $/ \mathrm{PTV})$ and homogeneity indices $\left(\mathrm{HI}=\mathrm{D}_{2 \%}-\mathrm{D}_{98 \%} / \mathrm{D}_{50 \%}\right)$ were 0.94 (range, 0.81-1.1) and 0.09 (range, 0.09-0.14), respectively.

\section{Dosimetric results of organs at risk}

Dosimetric parameters were obtained in percentages for simple interpretation. The dosimetric parameters in the brachytherapy and VMAT groups are listed in Table 4.

\section{Ipsilateral lung}

For ipsilateral lung, all the dosimetric parameters $\left(D_{\text {max }}, D_{0.1 c c}, D_{2 c c^{\prime}}, D_{\text {mean }}\right.$ and $\left.V_{10 \%}\right)$ were significantly higher for the VMAT group, except for $V_{5 \%}$. The $D_{0.1 c c}$ and $\mathrm{D}_{2 \mathrm{cc}}$ were almost 2-2.5 times higher in the VMAT group compared to the brachytherapy cohort.

\section{Contralateral lung}

For contralateral lung, all the dosimetric parameters were significantly lower for the brachytherapy group compared to the VMAT group. The mean lung dose in the brachytherapy group was $1.5 \%$ compared to $3.1 \%$ in the VMAT group ( $p$-value $<0.0001)$. $\mathrm{V}_{5 \%}$ and $\mathrm{V}_{10 \%}$ were close to zero in the brachytherapy group, while it was significantly higher in the VMAT cohort $(25 \%$ and $5.5 \%$, respec- tively, $p$-value $<0.0001)$. These parameters demonstrated better sparing of contralateral lung with brachytherapy.

\section{Contralateral breast}

Parameters collected included $\mathrm{D}_{0.1 \mathrm{c},}, \mathrm{D}_{2 \mathrm{cc}}, \mathrm{D}_{\text {mean }}, \mathrm{D}_{\max }$ and $\mathrm{V}_{5 \%}$. The $\mathrm{D}_{0.1 \mathrm{c} c^{\prime}}, \mathrm{D}_{2 \mathrm{cc}}$ and $\mathrm{D}_{\max }$ were significantly higher in the VMAT group ( $p$-value < 0.0001). Also, the mean dose was higher in the VMAT group (1.5\% vs. $1.7 \%)$, although not statistically significant. The volume receiving $5 \%$ of the prescribed dose $V_{5 \%}$ was significantly low in the brachytherapy group $(0.0 \%$ vs. $8.4 \%, p$-value $<0.0001)$.

\section{Ribs}

The high-dose received by ribs $\left(\mathrm{D}_{\max }, \mathrm{D}_{0.1 \mathrm{cc}}, \mathrm{D}_{1 \mathrm{cc}}\right.$ and $\left.\mathrm{D}_{2 \mathrm{cc}}\right)$ were significantly higher in the VMAT group ( $p$-value $<0.0001)$, probably because of the fact that PTV extending into the ribs was not cropped in the VMAT group, while CTV in the brachytherapy cohort was cropped from the ribs. The mean dose received by ipsilateral ribs was higher in the VMAT group (9.4\% vs. $8.1 \%)$, which was not statistically significant.

\section{Skin}

The skin was contoured as $5 \mathrm{~mm}$ thickness uniformly for all patients over the ipsilateral breast. Though, the maximum point dose was higher, $\mathrm{D}_{2 \mathrm{cc}}$ was significantly less with brachytherapy compared to VMAT technique ( $p$-value $<0.0001)$. There was no significant difference in the mean dose between the two groups.

\section{Heart}

The patients with left-sided breast cancer received higher doses to the heart when compared to right-sid- 
Table 4. Dosimetric parameters of organs at risk: volumetric-modulated arc therapy (VMAT) vs. brachytherapy Organ at risk

Parameter

Brachytherapy

VMAT

\begin{tabular}{|c|c|c|c|c|c|c|}
\hline & & Median (\%) & IQR (\%) & Median (\%) & IQR (\%) & \\
\hline \multirow[t]{6}{*}{ Ipsilateral lung } & $\mathrm{D}_{0.1 \mathrm{cc}}$ & 32.5 & $23.2-45.7$ & 86.1 & 73.7-93.0 & $<0.0001$ \\
\hline & $D_{2 c c}$ & 27.6 & 20.6-38.6 & 73.2 & 56.7-85.1 & $<0.0001$ \\
\hline & $\mathrm{D}_{\text {mean }}$ & 5.9 & $4.7-7.5$ & 10.5 & $7.8-12.8$ & 0.001 \\
\hline & $\mathrm{D}_{\max }$ & 34.1 & 24.4-50.9 & 90.3 & $82.4-95.6$ & $<0.0001$ \\
\hline & $V_{5 \%}$ & 37.3 & $30.4-56.8$ & 48.0 & $34.5-52.5$ & 0.641 \\
\hline & $V_{10 \%}$ & 13.5 & $8.3-22.2$ & 31.4 & $22.9-40.7$ & $<0.0001$ \\
\hline \multirow[t]{6}{*}{ Contralateral lung } & $\mathrm{D}_{0.1 \mathrm{icc}}$ & 5.0 & $2.4-7.9$ & 16.9 & $12.5-26.7$ & $<0.0001$ \\
\hline & $D_{2 c c}$ & 4.2 & $2.2-6.7$ & 14.5 & $11.3-23.4$ & $<0.0001$ \\
\hline & $\mathrm{D}_{\text {mean }}$ & 1.5 & $1.2-2.3$ & 3.1 & 2.1-5.0 & $<0.0001$ \\
\hline & $\mathrm{D}_{\max }$ & 5.3 & $2.6-8.4$ & 17.9 & $13.3-31.0$ & $<0.0001$ \\
\hline & $V_{5 \%}$ & 0.0 & $0.0-0.8$ & 25.0 & $16.4-34.5$ & $<0.0001$ \\
\hline & $V_{10 \%}$ & 0.0 & 0.0 & 5.5 & $1.0-17$ & $<0.0001$ \\
\hline \multirow[t]{6}{*}{ Contralateral breast } & $\mathrm{D}_{0.1 c c}$ & 4.7 & $2.8-8.2$ & 11.7 & 9.6-17.7 & $<0.0001$ \\
\hline & $\mathrm{D}_{1 c c}$ & 4.4 & $2.6-7.2$ & 10.2 & 8.1-14.8 & $<0.0001$ \\
\hline & $D_{2 c c}$ & 4.3 & $2.5-6.7$ & 9.6 & $7.5-13.7$ & $<0.0001$ \\
\hline & $\mathrm{D}_{\text {mean }}$ & 1.5 & $1.0-2.1$ & 1.7 & $1.0-3.0$ & 0.285 \\
\hline & $\mathrm{D}_{\max }$ & 4.9 & $3.0-9.0$ & 12.8 & $10.2-19.4$ & $<0.0001$ \\
\hline & $V_{5 \%}$ & 0.0 & $0.0-2.5$ & 8.4 & 4.6-23.9 & $<0.0001$ \\
\hline \multirow[t]{5}{*}{ Ribs } & $\mathrm{D}_{0.1 \mathrm{cc}}$ & 38.5 & 27.1-56.7 & 96.8 & 84.9-103.3 & $<0.0001$ \\
\hline & $\mathrm{D}_{1 c c}$ & 28.5 & $23.2-42.1$ & 74.5 & 59.2-91.5 & $<0.0001$ \\
\hline & $D_{2 c c}$ & 24.1 & 19.4-38.3 & 41.2 & 25.9-61.9 & $<0.0001$ \\
\hline & $\mathrm{D}_{\text {mean }}$ & 8.1 & $6.0-9.5$ & 9.4 & 7.0-12.4 & 0.091 \\
\hline & $\mathrm{D}_{\max }$ & 43.9 & $30.1-61.7$ & 103.3 & 97.4-106.1 & $<0.0001$ \\
\hline \multirow[t]{6}{*}{ Skin } & $\mathrm{D}_{0.1 \mathrm{ccc}}$ & 96.8 & 88.3-105.2 & 100.0 & 99.6-101.7 & 0.133 \\
\hline & $\mathrm{D}_{0.2 c c}$ & 93.5 & $85.8-102.8$ & 99.3 & $98.3-100.6$ & 0.017 \\
\hline & $D_{1 c c}$ & 82.7 & $78.2-86.3$ & 97.1 & $95.7-98.5$ & $<0.0001$ \\
\hline & $D_{2 c c}$ & 77.3 & 73.2-82.1 & 95.0 & 93-96.7 & $<0.0001$ \\
\hline & $\mathrm{D}_{\text {mean }}$ & 21.8 & 18.9-27.0 & 20.1 & $15.7-23.2$ & 0.052 \\
\hline & $\mathrm{D}_{\max }$ & 124.0 & $102.0-165.0$ & 104.3 & 102.6-105.1 & 0.004 \\
\hline \multirow[t]{7}{*}{ Heart doses for left-sided cases } & $\mathrm{D}_{0.1 \mathrm{cc}}$ & 24.1 & $17.2-32.0$ & 36.0 & 18.7-63.9 & 0.236 \\
\hline & $D_{2 c c}$ & 20.5 & $15.1-27.9$ & 30.2 & $15.2-51.9$ & 0.243 \\
\hline & $\mathrm{D}_{\text {mean }}$ & 5.9 & $5.3-9.5$ & 5.5 & 2.9-7.5 & 0.172 \\
\hline & $D_{\max }$ & 25.5 & $18.2-33.0$ & 39.2 & 19.7-68.0 & 0.199 \\
\hline & $V_{5 \%}$ & 51.0 & 40.8-84.7 & 26.3 & 21.6-63.4 & 0.024 \\
\hline & $V_{10 \%}$ & 11.4 & 7.1-35.1 & 18.2 & 5.0-21.1 & 0.937 \\
\hline & $V_{20 \%}$ & 0.5 & $0.0-4.9$ & 3.7 & $0.0-7.3$ & 0.196 \\
\hline \multirow[t]{7}{*}{ Heart doses for right-sided cases } & $\mathrm{D}_{0.1 \mathrm{cc}}$ & 7.1 & $6.0-14.7$ & 6.1 & $2.9-12.2$ & 0.369 \\
\hline & $D_{2 c c}$ & 6.5 & $5.4-12.6$ & 4.4 & $2.3-10.3$ & 0.165 \\
\hline & $\mathrm{D}_{\text {mean }}$ & 2.8 & $2.4-4.8$ & 0.85 & $0.6-1.8$ & 0.003 \\
\hline & $\mathrm{D}_{\max }$ & 7.4 & $6.3-15.8$ & 7.0 & $3.3-13.2$ & 0.513 \\
\hline & $V_{5 \%}$ & 5.3 & $1.8-35.0$ & 0.45 & $0.0-8.5$ & 0.14 \\
\hline & $V_{10 \%}$ & 0.0 & $0.0-4.3$ & 0.0 & $0.0-0.6$ & 0.909 \\
\hline & $V_{20 \%}$ & 0.0 & 0.0 & 0.0 & 0.0 & 1.0 \\
\hline \multirow[t]{6}{*}{ Ipsilateral non-target breast } & $\mathrm{D}_{\text {mean }}$ & 31.2 & $26.2-40.7$ & 20.0 & $16.3-27.5$ & $<0.0001$ \\
\hline & $V_{25 \%}$ & 41.7 & $33.0-52.7$ & 32.4 & $25.0-42.6$ & 0.008 \\
\hline & $V_{50 \%}$ & 19.5 & $14.0-26.8$ & 14.0 & $11.6-21.7$ & 0.125 \\
\hline & $V_{75 \%}$ & 7.0 & 5.3-10.9 & 6.3 & $5.0-8.8$ & 0.56 \\
\hline & $V_{90 \%}$ & 3.1 & $2.3-4.4$ & 2.4 & 2.1-3.9 & 0.204 \\
\hline & $V_{100 \%}$ & 2.5 & $1.3-2.8$ & 0.1 & $0.04-0.18$ & $<0.0001$ \\
\hline
\end{tabular}


ed cases. The dosimetric parameters, such as $\mathrm{D}_{0.1 \mathrm{cc}} \mathrm{D}_{2 \mathrm{cc}}$ $\mathrm{D}_{\text {max }}, \mathrm{V}_{10 \%}$, and $\mathrm{V}_{20 \%}$, were not significantly different between the two groups. There was also no significant difference in the mean dose received by the heart for left-sided (5.9\% vs. 5.5\%) cases. However, a significant difference was observed in the mean dose for right-sided cases $(2.8 \%$ vs. $0.8 \%, p$-value $=0.003)$.

\section{Ipsilateral non-target breast}

The ipsilateral breast tissue minus PTV was contoured. The mean dose was higher in the brachytherapy group $(31.2 \%$ vs. $20 \%$, $p$-value $<0.0001)$, which was statistically significant. $\mathrm{V}_{100 \%}$ was also higher in the brachytherapy group, and was statistically significant ( $2.5 \%$ vs. $0.1 \%, p$-value $<0.0001) . \mathrm{V}_{75 \%}(7 \%$ vs. $6.3 \%)$ and $\mathrm{V}_{50 \%}(19.5 \%$ vs. $14 \%)$ were not statistically significant. $\mathrm{V}_{25 \%}$ was significantly higher in the brachytherapy group $(41.7 \%$ vs. $32.4 \%$, $p$-value $=0.008)$

\section{Discussion}

Tumor bed boost irradiation is clinically relevant and significant in breast radiotherapy after conservative surgery, especially in younger patients (age $<50$ years) and those with an extensive intraductal component and grade 3 disease [8]. The two main types of radiation therapy, which could be applied to irradiate the tumor bed include external beam radiotherapy and brachytherapy. External beam radiotherapy may be delivered using fixed electron or photon beams, intensity-modulated radiation therapy, and volumetric-modulated arc therapy, while brachytherapy may be performed as interstitial procedure or as balloon brachytherapy. The present dosimetric study, to our knowledge, is the first study comparing dosimetric differences between interstitial brachytherapy and VMAT for tumor bed boost radiation in early-stage breast cancer. Even though Charaghvandi et al. [27] had dosimetrically compared interstitial brachytherapy and VMAT, their study was performed to evaluate the feasibility of single-fraction ablative radiotherapy in early-stage breast cancer, with brachytherapy plans generated virtually and with optimal placement of catheters. Our study findings revealed that interstitial brachytherapy results in significantly less dose to the contralateral breast, ipsilateral lung, contralateral lung, ribs, and skin, with similar heart-sparing in both the modalities, conforming outcomes of other studies. The reduction in most OARs doses could be explained by the fact that with interstitial brachytherapy, there is a rapid dose fall-off, whereas in VMAT, the doses were relatively higher, especially to the contralateral organs at risk due to scatter dose. In some cases, the medial entry point of VMAT beam was approaching the medial part of contralateral breast. The doses received by most of the OARs in the present study with brachytherapy were within the recommended dose-volume limits of ESTRO ACROP [28], as shown in Table 5.

Weed et al. [20] performed a dosimetric comparison for accelerated partial breast irradiation delivered using 3D-CRT, interstitial brachytherapy, and MammoSite brachytherapy, and found that $V_{20}$ and $V_{10}$ for ipsilateral lung was lower for brachytherapy techniques compared with 3D-CRT; heart-sparing was the same with all techniques. In the present study, though VMAT was used, $\mathrm{V}_{10}$ was still significantly less with interstitial brachytherapy. Additionally, no significant differences between the two modalities with regard to most dosimetric parameters of the heart for both left-sided and right-sided cases were observed.

Terheyden et al. [21] retrospectively evaluated 136 early-stage breast cancer patients, in whom doses to OARs after tumor bed boost irradiation were delivered with either high-dose-rate brachytherapy or 3D conformal radiation therapy. Patients treated with brachytherapy received a dose of $10 \mathrm{~Gy}$ in single-fraction, while those managed with external beam radiotherapy received a dose of 10 Gy in 5 fractions. The results demonstrated significant sparing of organs at risk with interstitial brachytherapy compared to 3D-CRT, with no significant difference for maximum dose to the heart for left-sided cases, which is analogous to the findings of the current study. The maximum dose to the heart with HDR-BT for left-sided cases and right-sided patients were $29.8 \%$ and $6 \%$, respectively, which is similar to the doses reported in the present study (25.5\% and $7.4 \%$ ). With 3D-CRT, the maximum doses for left-sided and right-sided irradiation were $29.9 \%$ and $16.7 \%$, respectively. However, in the present study where VMAT was used, $\mathrm{D}_{\text {max }}$ dose was lower for right-sided irradiation $(7 \%)$. Even though the $\mathrm{D}$ max dose was higher for left-sided irradiation $(39.2 \%)$, the $\mathrm{D}_{2 \mathrm{cc}}$ dose was $30.2 \%$. The maximum skin doses were higher, which may be related to the differences in skin delineation methods and location of tumors within the breast.

Lettmaier et al. [29] conducted a dosimetric study on left-sided breast cancer cases for radiation exposure to heart, lung, and skin between multicatheter interstitial brachytherapy-based accelerated partial breast irradiation and whole breast external beam radiotherapy. The EBRT technique used was 3D-CRT. When we com-

Table 5. Comparison of dose-volume of organs at risk for brachytherapy with ESTRO ACROP recommendations

\begin{tabular}{|c|c|c|c|}
\hline Organ at risk & Parameter & Recommended & $\begin{array}{l}\text { Present } \\
\text { study }\end{array}$ \\
\hline \multirow[t]{2}{*}{ Ipsilateral lung } & $\mathrm{D}_{\text {mean }}$ & $<8 \%$ & $5.9 \%$ \\
\hline & $\mathrm{D}_{0.1 \mathrm{cc}}$ & $<60 \%$ & $32.5 \%$ \\
\hline \multirow{2}{*}{$\begin{array}{l}\text { Heart for left- } \\
\text { sided cases }\end{array}$} & $\mathrm{D}_{\text {mean }}$ & $<8 \%$ & $5.9 \%$ \\
\hline & $\mathrm{D}_{0.1 c c}$ & $<50 \%$ & $24.1 \%$ \\
\hline \multirow[t]{2}{*}{ Rib } & $\mathrm{D}_{0.1 \mathrm{cc}}$ & $<90 \%$ & $38.5 \%$ \\
\hline & $\mathrm{D}_{1 \mathrm{cc}}$ & $<80 \%$ & $28.5 \%$ \\
\hline \multirow[t]{2}{*}{ Skin } & $D_{1 c c}$ & $<90 \%$ & $82.7 \%$ \\
\hline & $\mathrm{D}_{0.2 c c}$ & $<100 \%$ & $93.5 \%$ \\
\hline \multirow{2}{*}{$\begin{array}{l}\text { Ipsilateral } \\
\text { non-target breast }\end{array}$} & $V_{90}$ & $<10 \%$ & $3.1 \%$ \\
\hline & $V_{50}$ & $<40 \%$ & $19.5 \%$ \\
\hline $\begin{array}{l}\text { Contralateral } \\
\text { breast }\end{array}$ & $\mathrm{D}_{1 \mathrm{cc}}$ & No threshold & $4.4 \%$ \\
\hline
\end{tabular}


pared OARs dosimetric parameters to those in the present study, we found that the heart, $\mathrm{D}_{0.1 \mathrm{cc}}$ and $\mathrm{D}_{2 \mathrm{cc}}$ were similar in both studies $\left(\mathrm{D}_{0.1 \mathrm{cc}}: 24.1 \%\right.$ vs. $24.1 \%$; heart $\mathrm{D}_{2 \mathrm{cc}}$ : $21.4 \%$ vs. $20.5 \%$ ), and the ipsilateral lung $\mathrm{D}_{0.1 \mathrm{cc}}$ and $\mathrm{D}_{2 \mathrm{cc}}$ were relatively less $\left(\mathrm{D}_{0.1 \mathrm{cc}}: 39.2 \%\right.$ vs. $32.5 \% ; \mathrm{D}_{2 \mathrm{cc}}: 33.4 \%$ vs. $27.6 \%)$. The skin $\mathrm{D}_{0.1 \mathrm{cc}}$ and $\mathrm{D}_{2 \mathrm{cc}}$ were higher $\left(\mathrm{D}_{0.1 \mathrm{cc}}: 70.3 \%\right.$ vs. $96.8 \%$; $D_{2 c c}: 55.1 \%$ vs. $\left.77.3 \%\right)$. Although, the depth of tumor bed was not analyzed in the present study, the higher skin doses and lower lung dosimetric parameters might be attributed to the location of tumor cavity.

Major et al. [22] performed a dosimetric comparison for accelerated partial breast irradiation between multicatheter interstitial brachytherapy and intensity-modulated radiotherapy. They reported mean doses to lung as $5.1 \%$ vs. $7.1 \%$. Wu et al. [30] described a mean dose of $8 \%$ with intensity-modulated radiotherapy for accelerated partial breast irradiation. In our study, we found the mean lung doses as $5.9 \%$ vs. $10.5 \%$ confirming the dosimetric benefit of interstitial brachytherapy. The $\mathrm{D}_{0.1 \mathrm{cc}}$ and $\mathrm{D}_{2 \mathrm{cc}}$ in our study were $38.5 \%$ and $24.1 \%$ for interstitial brachytherapy, and $96.8 \%$ and $41.5 \%$ for VMAT, respectively. In the study by Major et al., the corresponding values for interstitial brachytherapy were $44.3 \%$ and $36.5 \%$, and $62.7 \%$ and $50.2 \%$ with IMRT. The dosimetric parameters for the ribs $\left(D_{0.1 \mathrm{cc}}=38.5 \%\right.$ and $\left.D_{1 \mathrm{cc}}=28.5 \%\right)$ in the present study were also in favor of interstitial brachytherapy, which is much lesser than that reported by Major et al. (58\% and $46 \%$ ) [22].

Therefore, there is significantly less dose to contralateral breast, ipsilateral lung, contralateral lung, ribs, and skin with interstitial brachytherapy, which could be advantageous in terms of toxicity comparing to VMAT, especially in elderly patients, given the possibility of implantation during surgery and thereby reducing overall duration of treatment $[31,32]$. Although, the present study is the first one to compare the dosimetric differences between interstitial brachytherapy and VMAT for tumor bed boost, it has several limitations. Firstly, it is retrospective in nature, which leads to inherent risk of bias. No specific inclusion and exclusion criteria were considered for choosing a particular modality of tumor bed boost for patients after whole breast radiotherapy. Electrons, a convenient modality for delivering tumor bed boost, were not included in this dosimetric analysis. Other factors, such as depth of the tumor bed, were not taken into account while selecting the boost technique. Further, this study presents only the dosimetric results, with no correlation to clinical end points, including acute and late toxicities, local control, and cosmetic outcomes.

\section{Conclusions}

High-dose-rate interstitial brachytherapy, when used as a modality for tumor bed boost irradiation with breast conservation therapy, results in significant reduction of dose to most organs at risk in comparison to VMAT. Prospective randomized controlled trial incorporating conformal fixed or intensity-modulated photon beams, electron beams, and interstitial brachytherapy, with correlation to clinical and patient-reported outcomes, are essential to identify the best treatment modality for delivering tumor bed boost.

\section{Disclosure}

The authors report no conflict of interest.

\section{References}

1. https://gco.iarc.fr/today/data/factsheets/populations/ 356-india-fact-sheets.pdf

2. Radiotherapy for early breast cancer. Cochrane Database Syst Rev 2002; 2: CD003647.

3. Fisher B, Anderson S, Bryant J et al. Twenty-year follow-up of a randomized trial comparing total mastectomy, lumpectomy, and lumpectomy plus irradiation for the treatment of invasive breast cancer. N Engl J Med 2002; 347: 1233-1241.

4. Whelan TJ, Olivotto IA, Parulekar WR et al. Regional nodal irradiation in early-stage breast cancer. N Engl J Med 2015; 373: 307-316.

5. Pass H, Vicini FA, Kestin LL et al. Changes in management techniques and patterns of disease recurrence over time in patients with breast carcinoma treated with breast-conserving therapy at a single institution. Cancer 2004; 101: 713-720.

6. Fodor J, Mozsa E, Zaka Z et al. Local relapse in young $(<$ or $=40$ years) women with breast cancer after mastectomy or breast conserving surgery: 15-year results. Magy Onkol 2005; 49: 203, 205-208.

7. Jalali R, Singh S, Budrukkar A. Techniques of tumour bed boost irradiation in breast conserving therapy: current evidence and suggested guidelines. Acta Oncologica 2007; 46: 879-892.

8. Bartelink H, Maingon P, Poortmans P et al. Whole-breast irradiation with or without a boost for patients treated with breast-conserving surgery for early breast cancer: 20-year follow-up of a randomised phase 3 trial. Lancet Oncol 2015; 16: $47-56$.

9. Hammer J, Track C, Seewald DH et al. 192-Iridium hdr boost in breast cancer treatment - experience from 644 patients (1984-1995). Radiother Oncol 2000; 55 (Suppl. 1): 32.

10. Polgar C, Fodor J, Orosz Z et al. Electron and high-dose-rate brachytherapy boost in the conservative treatment of stage I-II breast cancer first results of the randomized Budapest boost trial. Strahlenther Onkol 2002; 178: 615-623.

11. Hennequin C, Durdux C, Espie M et al. High-dose-rate brachytherapy for early breast cancer: an ambulatory technique. Int J Radiat Oncol Biol Phys 1999; 45: 85-90.

12. Manning MA, Arthur DW, Schmidt-Ullrich RK et al. Interstitial high-dose-rate brachytherapy boost: the feasibility and cosmetic outcome of a fractionated outpatient delivery scheme. Int J Radiat Oncol Biol Phys 2000; 48: 1301-1306.

13. Mansfield CM, Komarnicky LT, Schwartz GF et al. Ten-year results in 1070 patients with stages I and II breast cancer treated by conservative surgery and radiation therapy. Cancer 1995; 75: 2328-2336.

14. Hill-Kayser CE, Chacko D, Hwang WT et al. Long-term clinical and cosmetic outcomes after breast conservation treatment for women with early-stage breast carcinoma according to the type of breast boost. Int J Radiat Oncol Biol Phys 2011; 79: 1048-1054.

15. Kubaszewska M, Dymnicka M, Skowronek J et al. CT-image based conformal high-dose-rate brachytherapy boost in the conservative treatment of stage I-II breast cancer-introducing the procedure. Rep Pract Oncol Radiother 2008; 13: 227-239.

16. Dutta S, Sharma SC, Singh R et al. Quality assessment of interstitial implants in high- dose- rate brachytherapy after lumpectomy in patients of early stage breast cancer. Indian J Surg Oncol 2010; 1: 294-302. 
17. Aristei C, Palumbo I, Capezzali G et al. Outcome of a phase II prospective study on partial breast irradiation with interstitial multi-catheter high-dose-rate brachytherapy. Radiother Oncol 2013; 108: 236-241.

18. Aristei C, Maranzano E, Lancellotta V et al. Partial breast irradiation with interstitial multi-catheter high-dose-rate brachytherapy. Long-term results of a phase II prospective study. Radiother Oncol 2017; 124: 208-213.

19. Otto K. Volumetric modulated arc therapy: IMRT in a single gantry arc. Med Phys 2008; 35: 310-317.

20. Weed DW, Edmundson GK, Vicini FA et al. Accelerated partial breast irradiation: a dosimetric comparison of three different techniques. Brachytherapy 2005; 4: 121-129.

21. Terheyden MM, Melchert C, Kovács G. External beam boost versus interstitial high-dose-rate brachytherapy boost in the adjuvant radiotherapy following breast-conserving therapy in early-stage breast cancer: a dosimetric comparison. J Contemp Brachytherapy 2016; 8: 294-300.

22. Major T, Stelczer G, Pesznyák C et al. Multicatheter interstitial brachytherapy versus intensity modulated external beam therapy for accelerated partial breast irradiation: A comparative treatment planning study with respect to dosimetry of organs at risk. Radiother Oncol 2017; 122: 17-23.

23. Feng M, Moran JM, Koelling T et al. Development and validation of a heart atlas to study cardiac exposure to radiation following treatment for breast cancer. Int J Radiat Oncol Biol Phys 2011; 79: 10-18.

24. Ritter T, Quint DJ, Senan S et al. Consideration of dose limits for organs at risk of thoracic radiotherapy: atlas for lung, proximal bronchial tree, esophagus, spinal cord, ribs, and brachial plexus. Int J Radiat Oncol Biol Phys 2011; 81: 1442-1457.

25. Vicini F. National Surgical Adjuvant Breast and Bowel Project. A randomized phase III study of conventional whole breast irradiation (WBI) versus partial breast irradiation (PBI) for women with stage 0, I, or II breast cancer. NSABP PROTOCOL B-39-RTOG PROTOCOL. Pittsburgh, PA 2007; 413.

26. Major T, Gutiérrez C, Guix B et al. Recommendations from GEC ESTRO Breast Cancer Working Group (II): Target definition and target delineation for accelerated or boost partial breast irradiation using multicatheter interstitial brachytherapy after breast conserving open cavity surgery. Radiother Oncol 2016; 118: 199-204.

27. Charaghvandi RK, den Hartogh MD, van Ommen ALN et al MRI-guided single fraction ablative radiotherapy for early-stage breast cancer: a brachytherapy versus volumetric modulated arc therapy dosimetry study. Radiother Oncol 2015; 117: 477-482.

28. Strnad V, Major T, Polgar C et al. ESTRO-ACROP guideline: interstitial multi-catheter breast brachytherapy as accelerated partial breast irradiation alone or as boost-GEC-ESTRO Breast Cancer Working Group practical recommendations. Radiother Oncol 2018; 128: 411-420.

29. Lettmaier S, Kreppner S, Lotter M et al. Radiation exposure of the heart, lung and skin by radiation therapy for breast cancer: a dosimetric comparison between partial breast irradiation using multicatheter brachytherapy and whole breast teletherapy. Radiother Oncol 2011; 100: 189-194.

30. Wu S, He Z, Guo J et al. Dosimetric comparison of normal structures associated with accelerated partial breast irradiation and whole breast irradiation delivered by intensity modulated radiotherapy for early breast cancer after breast conserving surgery. Clin Transl Oncol 2014; 16: 69-76.

31. Tagliaferri L, Lancellotta V, Colloca G et al. Could a personalized strategy using accelerated partial breast irradiation be an advantage for elderly patients? A systematic review of the literature and multidisciplinary opinion. J Oncol 2020; 2020: 3928976.
32. Lancellotta V, Kovács G, Tagliaferri L et al. Age is not a limiting factor in interventional radiotherapy (brachytherapy) for patients with localized cancer. Biomed Res Int 2018; 2018: 2178469. 